\title{
Quantitative Proteomics Reveals the Regulatory Networks of Circular RNA BTBD7_hsa_circ_000563 in human coronary artery
}

\section{Jia-Xin Chen}

Jiangsu Province Hospital and Nanjing Medical University First Affiliated Hospital

\section{Lei Hua}

Jiangsu Province Hospital and Nanjing Medical University First Affiliated Hospital

\section{Chen-Hui Zhao}

Jiangsu Province Hospital and Nanjing Medical University First Affiliated Hospital

Qiao-Wei Jia

Jiangsu Province Hospital and Nanjing Medical University First Affiliated Hospital

Jing Zhang

Jiangsu Province Hospital and Nanjing Medical University First Affiliated Hospital

Jin-Xia Yuan

Jiangsu Province Hospital and Nanjing Medical University First Affiliated Hospital

Yong-Jie Zhang

Nanjing Medical University

Jian-Liang Jin

Nanjing Medical University

Mu-Feng Gu

Nanjing Medical University

\section{Zhi-Yuan Mao}

Nanjing Medical University

Hai-Jian Sun

Nanjing Medical University

\section{Lian-Sheng Wang}

Jiangsu Province Hospital and Nanjing Medical University First Affiliated Hospital

\section{Wen-Zhu Ma}

Jiangsu Province Hospital and Nanjing Medical University First Affiliated Hospital

Enzhi Jia ( $\sim$ enzhijia@njmu.edu.cn )

The First Affiliated Hospital of Nanjing Medical University https://orcid.org/0000-0003-1354-9855

\section{Research article}

Keywords: Coronary heart disease, circRNA, BTBD7_hsa_circ_000563, Coronary artery segment 
Posted Date: March 6th, 2020

DOI: https://doi.org/10.21203/rs.3.rs-16281/v1

License: (a) (i) This work is licensed under a Creative Commons Attribution 4.0 International License. Read Full License 


\section{Abstract}

Background: To investigate the association of BTBD7_hsa_circ_000563 expression in coronary artery segments with atherosclerotic stenosis, and to explore the proteome-wide identification of the BTBD7_hsa_circ_000563-regulated proteins in coronary artery

Methods: The coronary artery samples were obtained from two autopsy cases. The epicardial coronary artery of every autopsy was divided into 10 segments, and coronary atherosclerosis grade and extent of the coronary artery segments were analysed by Haematoxylin and Eosin (H\&E) staining. The BTBD7_hsa_circ_000563 expression of 8 segments from case 2 was quantified using RT-qPCR analysis.

Results: The present study demonstrated that coronary artery segments with severe atherosclerotic stenosis showed extremely low expression of the BTBD7_hsa_circ_000563, compared with normal coronary artery segments. Furthermore, it was predicted that hsa-miR-155-5p, and hsa-miR-130a-3p are targets of the BTBD7_hsa_circ_000563. The results from the present study may laid an epigenetic foundation for studying the underlying mechanisms of the development and progression of coronary artery atherosclerosis.

Conclusions: BTBD7_hsa_circ_000563 were involved in atherosclerotic changes in coronary artery segments of human being, and the verification study, mechanism study, and function study are necessary in order for CAD patients to benefit from the personalized medicine in the future.

\section{Background}

Cardiovascular disease (CVD), especially coronary artery disease (CAD) arising from atherosclerosis is the leading cause of human morbidity and mortality across the world [1]. Although there are imaging modalities and serological indicators of estimating the extent of atherosclerosis in affected subjects, current established measurements used in the monitoring of CADs are concentrated on the late symptomatic phases. Therefore, in order for early detection and timely therapeutic intervention of CAD, there remains an urgent need for biomarkers exploring. Increasing evidence has implicated the circRNAs in the pathogenesis of CAD [2-3]. Among them, BTBD7_hsa_circ_000563 (also known as hsa_circ_000847) stands out as a clinically relevant tissue-associated circRNAs [4] and is involved in human various diseases [5-6].

In this study, reverse transcription (RT) followed by real-time quantitative (q) PCR (RT-qPCR) detection of BTBD7_hsa_circ_000563 was employed to discovery and validation of candidate biomarkers of CAD in coronary artery, sampled from subjects with coronary artery disease. And, in our present study, quantitative proteomics-based strategies were employed to identify BTBD7_hsa_circ_000563-regulated proteins in human coronary artery.

\section{Methods}

\section{Study subjects}


The coronary artery samples were obtained from two autopsy cases at department of human anatomy in Nanjing Medical University. Informed consent from the bereaved family was obtained for the research use only of samples and the autopsy was conducted according to the guideline of the university. The methods were performed in accordance with the approved guidelines, and all experimental protocols were approved by the ethics committee of the Nanjing Medical University and the First Affiliated Hospital of Nanjing Medical University. The age was 64 and 68 years respectively, and the female/male ratio 0:2. The postmortem delay varied between 1 and 2 days. At autopsy, epicardial coronary arteries were removed from the hearts. The epicardial coronary artery of every autopsy was divided into 10 segments: the proximal segment, the midsegment, the distal segment of the left anterior descending (LAD), left circumflex (LCX), right coronary artery (RCA) respectively, and the left main trunk (LM). And, every coronary artery segment was divided into three groups: RNA group, protein group and pathological group. The segments at the RNA group and protein group were snap-frozen in liquid nitrogen and stored at $-80^{\circ} \mathrm{C}$ for real-time PCR analysis. In addition, the segments at the pathological group were fixed overnight in $10 \%$ formalin and embedded in paraffin for histological analysis.

\section{Pathological analysis}

First, the group was collected in EDTA decalcification fluid for about two weeks. Then the pathological coronary artery segments were fixed overnight in $10 \%$ formalin and processed for paraffin embedding: longitudinal 5 micrometer thick consecutive sections were obtained by a rotarymicrotome (Leica RM2235, Leica Biosystems Nussloch GmbH, Heidelberger Str. 17 - 19D-69226 Nussloch, Germany) and stained with Haematoxylin and Eosin (H\&E) in order to observe and morphometrically. Each slide was examined by steromicroscope 10X (Leica DM2500 Wien, Austria) at 5x to 20x original magnification and digitized by a image analysis system was used (Leica LAS, Wetzlar, Germany), as Mingyue Ji, Jinxia Yuan ect. reported in 2019.

Coronary atherosclerotic grading and extent of the segments were indepently analysed by pathologists committed to making a connection of current histological grading according to American Heart Association (AHA) classification guidelines [7].

\section{Selection of circular RNAs}

Based on our previous studies, significant differences between CAD and non-CAD subjects with respect to miR-221 ( $p=0.001)$, miR-155 ( $p=0.049)$, and miR-130a ( $p=0.001)$ were found [8]. We selected hsa-miR-221$3 p$, hsa-miR-155-5p, and hsa-miR-130a-3p which were detected on the qPCR validation for the joint analysis of miRNA and circRNAs profiling data. We used miRNA target predictions based on Starbase v2.0 (http://starbase.sysu.edu.cn/mirCircRNA.php). The interactions between hsa-miR-155-5p, hsa-miR-130a-3p and BTBD7_hsa_circ_000563 was found respectively. Therefore, the BTBD7_hsa_circ_000563 was selected for the target gene in the present study.

\section{RNA extraction and RT-qPCR}

Total RNA was extracted from the coronary artery segments using Trizol (15596018,Invitrogen) following the manufacturer's instructions and checked for a RIN number by an Agilent Bioanalyzer 2100 (Agilent 
technologies, US).

Total RNA $(1 \mu \mathrm{g})$ was used as a template to prepare cDNA before PCR as reported by Ren-you Pan etc al.,in 2019.The BTBD7_hsa_circ_000563 expression was quantified using SYBR Green Realtime PCR Master Mix (TOYOBO, QPK-212,Japan) on the ABI 7900HT squence detection system (Applied Biosystems,7900HTLUSA)[9].

\section{Protein extraction, LC-MS/MS Analysis}

For the quantification of the protein, $500 \mu \mathrm{g}$ of total protein was extracted using a multi-step L3 lysis buffer/M2 lysis buffer protocol, as Ya-qing Zhou, Jinxia Yuan ect. reported in 2019. First, the Bradford Method was used for protein quantification. We took $100 \mu \mathrm{g}$ protein and dissolve it to $1 \mu \mathrm{g} / \mu \mathrm{L}$ using M2 lysis buffer (without thiourea). It was diluted 6-fold by adding 5 volumes of $100 \mathrm{mM}$ TEAB before shaking by centrifugation. Substantially,the protein samples were digested overnight at $37^{\circ} \mathrm{C}$ by adding $2 \mu \mathrm{g}$ trypsin. Next, we weighed $10 \mathrm{mg}$ of $\mathrm{C} 18$ column for each peptide sample. With discarding the supernatant by centrifugation,the sample was washed with $0.1 \% \mathrm{FA}+3 \% \mathrm{ACN}$ twice for desalting and eluted with $1 \mathrm{~mL}$ $0.1 \% \mathrm{FA}+80 \% \mathrm{ACN}$. The eluted peptides were dried with a vacuum concentration meter. Third, the peptide sample was diluted to $1 \mu \mathrm{g} / \mu \mathrm{l}$ on the peptide sample, and the scanning mode was set to $5 \mu \mathrm{l}$ and $120 \mathrm{~min}$. The peptides in the sample with a mass-to-charge ratio of 350-1500 were scanned. The mass spectrometry data was collected using the Triple TOF 5600 + LC/MS system (AB SCIEX,USA). For IDA (Information Dependent Acquisition), a first-order mass spectrum was scanned with an ion accumulation time of $250 \mathrm{~ms}$, and a secondary mass spectrum of 30 precursor ions was acquired with an ion accumulation time of $50 \mathrm{~ms}$. The MS1 spectrum was acquired in the range of $350-1200 \mathrm{~m} / \mathrm{z}$, and the MS2 spectrum was acquired in the range of $100-1500 \mathrm{~m} / \mathrm{z}$. Set the precursor ion dynamic exclusion time to $15 \mathrm{~s}$.

\section{Data analysis}

We performed protein identification and quantification by ProteinPilot software (https://sciex.com.cn/products/software/proteinpilot-software, version 4.5, SCIEX, Redwood City, California, USA). The MS/MS spectrum was searched for the uniprot-Homo_sapiens protein database. To identify peptides, the Paragon algorithm was employed against the uniprot-Homo_sapiens database. The parameters were set as follows: cysteine modified with iodoacetamide; biological modifications were selected as ID focus. For false discovery rate (FDR) calculation, an automatic decoy database search strategy was employed to estimate FDR using the PSPEP (Proteomics System Performance Evaluation Pipeline Software, integrated in the ProteinPilot Software). Only unique peptides with global FDR values from fit less than $1 \%$ were considered for further analysis. Skyline v4.2 software was applied for MS1 filtering and ion chromatogram extractions for peptides label-free quantification, the parameters setting for skyline MS1 filtering were as the same as. With the results of the Skyline quantification, the mean value of the ratio of each group was used to calculate the fold change.

Owing to analysis need, the eight samples were divided into four groups according to the ascending order of BTBD7_hsa_circ_000563 expression level, functioning as a comparative basis for further analysis of proteome data. After grouping eight samples according to BTBD7_hsa_circ_000563 expression level, the median quantitative value of the samples in each group was taken as the expression value of a protein in 
that group, so a new expression value of BTBD7_hsa_circ_000563 was obtained in each group. Next, the comparison between groups (finding the fold change) is also compared with the new expression value of each group. In order to test whether the difference between the groups was significant, the T test was carried out and the p-value was obtained. The screening criteria of significant difference proteins were as follows: significantly differentially expressed with a threshold fold change over 1.5 or less than 0.667 between groups; $\mathrm{P}<0.05$ in the comparison between groups; of the three comparison groups, at least one of them satisfies the above criteria with ratio trend of increasing or decreasing among all the three comparison groups following the increasing of the BTBD7_hsa_circ_000563 expression level.

\section{Bioinformatics and Annotations}

To determine the biological and functional properties of all identified proteins, the Gene Ontology Terms (http://geneontology.org/) were used to describe the properties of genes and gene products in organisms. To this end, homology searches were first performed on all identified sequences using the local NCBI blastp program for the NCBInr animal database. The e value was set to be less than $1 \mathrm{e}-5$, and the best hit for each query sequence took into account the $\mathrm{GO}$ term match.Functional annotation of genes from the new genome and studies of genome evolution were investigated using the orthologous protein system population (COG, http://www.ncbi.nlm.nih.gov/COG/). KEGG is the main public database on Pathway (Kanehisa, 2008), and Pathway analysis identifies the most important biochemical metabolic pathways and signal transduction pathways involved in protein. To identify candidate biomarkers, we used hypergeometric testing for GO enrichment and KEGG pathway enrichment. The protein-protein interaction (PPI) network of BTBD7_hsa_circ_000563-regulated proteins was performed by means of the Search Tool for the Retrieval of Interacting Genes/Proteins database (STRING) v10.0 [10] following default settings and visualized using Cytoscape v3.2.1 (http://www.cytoscape.org).

\section{Results}

Natural history and histological classification of atherosclerotic lesions of the coronary artery samples

The natural history and histological classification of atherosclerotic lesions of the coronary artery samples which were analyzed by Haematoxylin and Eosin (H\&E) staining was shown in Table 1. 
Table 1

Natural history and histological classification of atherosclerotic lesions of the coronary artery samples

\begin{tabular}{|c|c|c|c|c|c|c|c|c|c|c|c|c|}
\hline Case1 & Age & Gender & LM & $\begin{array}{l}\text { LAD- } \\
p\end{array}$ & $\begin{array}{l}\text { LAD- } \\
\mathrm{m}\end{array}$ & $\begin{array}{l}\text { LAD- } \\
\text { d }\end{array}$ & $\begin{array}{l}\text { LCX- } \\
\text { p }\end{array}$ & $\begin{array}{l}\text { LCX- } \\
\text { m }\end{array}$ & $\begin{array}{l}\text { LCX- } \\
\text { d }\end{array}$ & $\begin{array}{l}\text { RCA- } \\
p\end{array}$ & $\begin{array}{l}\text { RCA- } \\
\text { m }\end{array}$ & $\begin{array}{l}\text { RCA- } \\
\text { d }\end{array}$ \\
\hline Grade & 64 & Male & 3 & 4 & 3 & 4 & 4 & 4 & 3 & 4 & 4 & 4 \\
\hline Stage & & & 4 & 3 & 3 & 3 & 4 & 3 & 3 & 4 & 3 & 3 \\
\hline Case2 & Age & Gender & LM & $\begin{array}{l}\text { LAD- } \\
\mathrm{p}\end{array}$ & $\begin{array}{l}\text { LAD- } \\
\mathrm{m}\end{array}$ & $\begin{array}{l}\text { LAD- } \\
\text { d }\end{array}$ & $\begin{array}{l}\mathrm{LCX}- \\
\mathrm{p}\end{array}$ & $\begin{array}{l}\mathrm{LCX}- \\
\mathrm{m}\end{array}$ & $\begin{array}{l}\text { LCX- } \\
\text { d }\end{array}$ & $\begin{array}{l}\text { RCA- } \\
p\end{array}$ & $\begin{array}{l}\text { RCA- } \\
\mathrm{m}\end{array}$ & $\begin{array}{l}\text { RCA- } \\
\text { d }\end{array}$ \\
\hline Grade & 68 & Male & 3 & 3 & 3 & 1 & 1 & 1 & 1 & 1 & 3 & 1 \\
\hline Stage & & & 3 & 3 & 3 & 0 & 0 & 1 & 1 & 2 & 3 & 1 \\
\hline \multicolumn{13}{|c|}{$\begin{array}{l}\text { LM, the left main trunk; LAD-p, proximal segment of the left anterior descending; LAD-m, midsegment of } \\
\text { the left anterior descending; LAD-d, distal segment of the left anterior descending; LCX-p, proximal } \\
\text { segment of the left circumflex; LCX-m, midsegment of the left circumflex; LCX-d, distal segment of the } \\
\text { left circumflex; RCA-p, proximal segment of the right coronary artery; RCA-m, midsegment of the right } \\
\text { coronary artery; RCA-d, distal segment of theright coronary artery; }\end{array}$} \\
\hline \multicolumn{13}{|c|}{$\begin{array}{l}\text { Grade, } 1 \text { : stenosis from } 0 \text { to } 25 \% ; \text {;Grade, } 2 \text { :stenosis from } 26 \text { to } 50 \% \text {;Grade, } 3 \text { : stenosis from } 51 \text { to } \\
75 \% \text {;Grade, } 4 \text { :stenosis from } 76 \text { to } 100 \%\end{array}$} \\
\hline
\end{tabular}

The analysis of Haematoxylin and Eosin (H\&E) staining indicated that atherosclerotic lesions were found at the segments of coronary artery in case 1 and case 2. However, coronary atherosclerosis grade and extent in the case 1 was more serious than that in case 2 . All of the examined coronary segments in case 1 showed atherosclerotic changes of the intima, ranging from lesions classifiable as atherosclerotic tunica intima to secondary affection tunica intima, and stenosis from 51 to $100 \%$. Conversely, atherosclerotic changes of normal tunica intima, fatty streak tunica intima, fibrous plaques tunica intima, and atherosclerotic tunica intima were found at coronary segments in case 2 with coronary stenosis from 0 to $75 \%$.

\section{The BTBD7_hsa_circ_000563 expression in coronary artery segments}

Due to the RNA degradation, the purity and concentration of the total RNA at the all coronary artery segments in case 1 and at the midsegment and distal segment of the right coronary artery in case 2 failed to meet standards for RT-qPCR analysis. Therefore, the BTBD7_hsa_circ_000563 expression via RT-qPCR has been detected in coronary artery samples from case 2 except the midsegment and distal segment of the right coronary artery.

In the RT-qPCR assay, BTBD7_hsa_circ_000563 expression was assessed and the results revealed that the expression of BTBD7_hsa_circ_000563 were higher in normal coronary artery segments, by comparison 
with that in coronary artery segments with severe atherosclerosis, and the results were shown in Table 2 and Fig. 1. From the autopsy case 2 , in coronary artery segment of proximal segment of the left circumflex with normal tunica intim and stenosis from 0 to 25\%,BTBD7_hsa_circ_000563 expression was most significantly increased by 20 -fold compared with that in the left main trunk with atherosclerotic tunica intima and stenosis from 51 to $75 \%$. 
Table 2

The BTBD7_hsa_circ_000563 expression in coronary artery segments

\begin{tabular}{|c|c|c|c|c|c|c|}
\hline Segment & h-actin & ct & $\Delta \mathrm{ct}$ & $\Delta \Delta \mathrm{ct}$ & $2^{\wedge}-\Delta \Delta \mathrm{ct}$ & Expression level \\
\hline \multirow[t]{3}{*}{ LM } & 20.15 & 33.85 & 13.7 & 2.08 & 0.236514412 & \multirow[t]{3}{*}{0.1705} \\
\hline & 20.16 & 35.04 & 14.88 & 3.26 & 0.10438599 & \\
\hline & 20.18 & 31.87 & 11.69 & 0.07 & 0.952637998 & \\
\hline \multirow[t]{3}{*}{ LAD-p } & 20.06 & 31.41 & 11.35 & -0.27 & 1.205807828 & \multirow[t]{3}{*}{0.013726} \\
\hline & 19.85 & 37.38 & 17.53 & 5.91 & 0.016630784 & \\
\hline & 20.06 & 38.21 & 18.15 & 6.53 & 0.010821168 & \\
\hline \multirow[t]{3}{*}{ LAD-m } & 20.17 & 37.29 & 17.12 & 5.5 & 0.022097087 & \multirow[t]{3}{*}{1.325879} \\
\hline & 20.17 & 31.46 & 11.29 & -0.33 & 1.257013375 & \\
\hline & 20.19 & 31.33 & 11.14 & -0.48 & 1.394743666 & \\
\hline \multirow[t]{3}{*}{ LAD-d } & 19.14 & 30.86 & 11.72 & 0 & 1 & \multirow[t]{3}{*}{1} \\
\hline & 19.17 & 29.8 & 10.63 & 0 & 1 & \\
\hline & 18.91 & 31.42 & 12.51 & 0 & 1 & \\
\hline \multirow[t]{3}{*}{ LCX-p } & 18.85 & 28.19 & 9.34 & -2.28 & 4.856779538 & \multirow[t]{3}{*}{3.99756} \\
\hline & 18.98 & 30.86 & 11.88 & 0.26 & 0.835087919 & \\
\hline & 19.07 & 29.04 & 9.97 & -1.65 & 3.138336392 & \\
\hline \multirow[t]{3}{*}{ LCX-d } & 20.18 & 29.35 & 9.17 & -2.45 & 5.464161027 & \multirow[t]{3}{*}{5.31411} \\
\hline & 20.11 & 30.09 & 9.98 & -1.64 & 3.116658319 & \\
\hline & 20.26 & 29 & 8.74 & -2.88 & 7.361501205 & \\
\hline \multirow[t]{3}{*}{ RCA-p } & 20.11 & 30.08 & 9.97 & -1.65 & 3.138336392 & \multirow[t]{3}{*}{2.28259} \\
\hline & 19.94 & 30.5 & 10.56 & -1.06 & 2.084931522 & \\
\hline & 20.02 & 30.94 & 10.92 & -0.7 & 1.624504793 & \\
\hline \multirow[t]{3}{*}{ LCX-m } & 20.39 & 34.04 & 13.65 & 2.03 & 0.244855074 & \multirow[t]{3}{*}{2.15609} \\
\hline & 20.38 & 31.45 & 11.07 & -0.55 & 1.464085696 & \\
\hline & 20.22 & 30.33 & 10.11 & -1.51 & 2.848100391 & \\
\hline $\begin{array}{l}\text { LM, the lef } \\
\text { the left an } \\
\text { segment o } \\
\text { left circum }\end{array}$ & $\begin{array}{l}\text { left cir } \\
\text { RCA-p, }\end{array}$ & $\begin{array}{l}\text { ex; LCX } \\
\text { nal se }\end{array}$ & $\begin{array}{l}\text { al segm } \\
\text { tal segr } \\
\text { midseg } \\
\text { nt of th }\end{array}$ & $\begin{array}{l}\text { of the le } \\
\text { t of the } \\
\text { it of the } \\
\text { ht coror }\end{array}$ & $\begin{array}{l}\text { nterior descend } \\
\text { anterior descen } \\
\text { circumflex; LC〉 } \\
\text { artery }\end{array}$ & $\begin{array}{l}\text { AD-m, midsegment } \\
\text { LCX-p, proximal } \\
\text { stal segment of the }\end{array}$ \\
\hline
\end{tabular}




\section{The screening of significant difference proteins according to the BTBD7_hsa_circ_000563 expression level}

The identification and quantification of significant difference proteins according to the BTBD7_hsa_circ_000563 expression level are presented in Table 3. 
Table 3

The screening of significant difference proteins according to the BTBD7_hsa_circ_000563 expression level

\begin{tabular}{|c|c|c|c|}
\hline Protein ID & Express trend & Protein ID & Express trend \\
\hline sp|P00367|DHE3_HUMAN & UP & sp|A0A075B6I0|LV861_HUMAN & DOWN \\
\hline sp|Q92552|RT27_HUMAN & UP & sp|P07954|FUMH_HUMAN & UP \\
\hline sp|P13861|KAP2_HUMAN & UP & sp|Q02985|FHR3_HUMAN & DOWN \\
\hline sp|075746|CMC1_HUMAN & UP & sp|Q9HA77|SYCM_HUMAN & UP \\
\hline sp|Q9UJY1|HSPB8_HUMAN & UP & sp|075208|COQ9_HUMAN & UP \\
\hline sp|P48735|IDHP_HUMAN & UP & sp|Q9UM22|EPDR1_HUMAN & UP \\
\hline sp|096000|NDUBA_HUMAN & UP & sp|Q9Y6W5|WASF2_HUMAN & UP \\
\hline sp|P13671|CO6_HUMAN & DOWN & sp|P09622|DLDH_HUMAN & UP \\
\hline sp|Q99733|NP1L4_HUMAN & UP & sp|P48681|NEST_HUMAN & UP \\
\hline sp|P01624|KV315_HUMAN & DOWN & sp|Q16836|HCDH_HUMAN & UP \\
\hline sp|Q9Y235|ABEC2_HUMAN & UP & sp|P01700|LV147_HUMAN & DOWN \\
\hline sp|P16615|AT2A2_HUMAN & UP & sp|P36542|ATPG_HUMAN & UP \\
\hline sp|P02760|AMBP_HUMAN & DOWN & sp|P06312|KV401_HUMAN & DOWN \\
\hline sp|P19022|CADH2_HUMAN & UP & sp|Q9NRPO|OSTC_HUMAN & UP \\
\hline sp|P61626|LYSC_HUMAN & DOWN & sp|P01701|LV151_HUMAN & DOWN \\
\hline sp|043837|IDH3B_HUMAN & UP & sp|P02511|CRYAB_HUMAN & UP \\
\hline sp|Q9H479|FN3K_HUMAN & UP & sp|Q15628|TRADD_HUMAN & UP \\
\hline sp|095831|AIFM1_HUMAN & UP & sp|P23786|CPT2_HUMAN & UP \\
\hline sp|P17540|KCRS_HUMAN & UP & sp|P00846|ATP6_HUMAN & UP \\
\hline sp|P21912|SDHB_HUMAN & UP & sp|075367|H2AY_HUMAN & UP \\
\hline sp|P50213|IDH3A_HUMAN & UP & sp|P12235|ADT1_HUMAN & UP \\
\hline sp|P26440|IVD_HUMAN & UP & sp|P08559|ODPA_HUMAN & UP \\
\hline sp|Q9Y287|ITM2B_HUMAN & DOWN & sp|P42765|THIM_HUMAN & UP \\
\hline sp|Q99798|ACON_HUMAN & UP & sp|P30046|DOPD_HUMAN & UP \\
\hline sp|P46777|RL5_HUMAN & UP & & \\
\hline Figure 1 & & & \\
\hline
\end{tabular}


According to these analysis, 49 proteins were differentially abundant between the BTBD7_hsa_circ_000563 expression level groups. Among the 49 proteins, 10 proteins were found down-regulated, while 39 were determined to be up-regulated with the increasing of the BTBD7_hsa_circ_000563 expression level.

\section{GO annotation of the Differentially Expressed Proteins}

To further determine the biological and functional properties of all differentially expressed proteins, the GO annotation was employed to analyze the differentially expressed proteins, and the annotation results were shown in Fig. 2.

GO annotation with respect to the 49 differentially expressed proteins in human coronary artery samples suggested that the cellular component of these proteins was predominantly derived from the mitochondrial matrix (G0:0005759), and mitochondrial inner membrane (GO:0005743)(Fig. 2A). Moreover, the molecular function of these proteins primarily included NAD or NADH binding, 3 iron, 4 sulfur cluster binding, FAD binding, NAD binding, and isocitrate dehydrogenase (NAD+) activity (Fig. 2B). In addition, the biological process of these proteins participating in predominantly included the respiratory electron transport chain (GO: 0022904), tricarboxylic acid cycle (G0:0006099), and mitochondrial electron transport, NADH to ubiquinone (G0:0006120)(Fig. 2C).

\section{KEGG enrichment of the Differentially Expressed Proteins}

KEGG pathways enrichment analysis was performed and that BTBD7_hsa_circ_000563-related proteins were associated with the pathways including Parkinson's disease, Alzheimer's disease, Fatty acid metabolism, Primary immunodeficiency, oxidative phosphorylation, Huntington's disease, D-Glutamine and D-glutamate metabolism, Valine, leucine and isoleucine degradation, Fatty acid elongation in mitochondria, metabolic pathways, Apoptosis, microbial metabolism in diverse environments, and citrate cycle (TCA cycle) were observed (Fig. 3).

\section{Protein - protein interaction of the Differentially Expressed Proteins}

The protein - protein interaction of the BTBD7_hsa_circ_000563-regulated proteins was generated by means of the STRINTG database. 25 of the 49 differentially expressed proteins are engaged in a PPI network based on STRING database Besides, the network contains 30 nodes and 86 edges, and the 30 proteins joining in the PPI networks were categorized into several functional groups. Therefore, the PPI networks indicated that the BTBD7_hsa_circ_000563-regulated proteins have potential to interact with each other and work in union to exercise their functions. Moreover, to evaluate the classification of the BTBD7_hsa_circ_000563-regulated proteins, we used the classification system to sort out the proteins. The 49 proteins hit by the classification system were divided into several classes (Fig. 4). Taking these results together, BTBD7_hsa_circ_000563-regulated proteins may make a difference to various biological processes particularly in regulating dehydrogenase and ATP synthase.

\section{Discussion}


In the study,we foud that BTBD7_hsa_circ_000563 were involved in atherosclerotic changes in human being coronary artery segments. And, the expression levels of BTBD7_hsa_circ_000563 were higher in relatively normal coronary artery segments, as compared with in coronary artery segments with severe atherosclerosis. In addition, by means of a proteomics approach, 49 proteins regulated by enhanced BTBD7_hsa_circ_000563 expression were identified in human coronary artery segments. Among these 49 proteins, none of them have been identified as BTBD7_hsa_circ_000563 regulated target protein. Therefore, the results from the present study provides an abundant source for the functional studies of BTBD7_hsa_circ_000563 in coronary artery.

BTBD7_hsa_circ_000563 is located on chromosome 14, and the gene coding starts from position 93,760,203 and ends on position 93,762,503 of chromosome 14 (https://circinteractome.nia.nih.gov/bin/circsearch?circrna=hsa_circ_0000563), and the BTBD7_hsa_circ_000563 was first reported by Memczak et al [5]. Afterwards, significantly different expression of the BTBD7_hsa_circ_000563 was found at human cells (H1hesc, Nhek, and Ag04450) [11], human different brain regions [6], and human endothelial progenitor cells [4] via RNA Sequencing analysis. However, the present study demonstrated that coronary artery segments with severe atherosclerotic stenosis showed extremely low expression of the BTBD7_hsa_circ_000563, as compared with normal coronary artery segments. Furthermore, it was predicted that hsa-miR-155-5p, and hsa-miR-130a-3p are targets of the BTBD7_hsa_circ_000563. The above results of the present study laid an epigenetic foundation for studying the underlying mechanisms of the development of coronary artery atherosclerosis. However, the proteome-wide analysis of BTBD7_hsa_circ_000563-regulated protein in coronary artery has not been reported.

To further explore the foundation and mechanism underlying the association between the BTBD7_hsa_circ_000563 and coronary artery atherosclerosis. The proteome-wide analysis was conducted in the present study. The proteomics analysis identified 49 proteins regulated by BTBD7_hsa_circ_000563 overexpression in human coronary artery segments. And, bioinformatic analyses revealed that a large number of differentially expressed proteins were located in mitochondrion and involved in citrate cycle (TCA cycle) pathway, and the ATP synthase may be the hub of the regulatory networks of BTBD7_hsa_circ_000563 in coronary artery.

As complex dynamic organelles, mitochondria play numerous functions pertaining to cellular metabolism and homoeostasis, the hallmark of mitochondria is cellular energy generation by means of oxidative phosphorylation (OXPHOS) [12-13]. And, the mitochondrial DNA encodes only 13 OXPHOS proteins; the remaining about 1500 proteins from the mitochondrial proteome are transferred to the mitochondria and are encoded in the nuclear genome[14]. In addition, several converging metabolic pathways including the folate metabolism, TCA cycle, and sulphur metabolism consist in whole or partial components of the mitochondria house [15]】

Coronary artery disease has complex etiology, and mitochondrial dysfunction exerts influence on various celluar aberrations including energy deficit, deregulation of autophagy, metabolic abnormalities, excessive production of reactive species, endoplasmic reticulum stress, and activation of apoptosis. The impairment 
of the ATP synthesis and respiratory chain have been considered as a key of mitochondrial dysfunction. Therefore, recovering of the mitochondrial function including respiratory activity and ATP-producing capacity have been considered as a primary therapeutic target to improve the prognosis of coronary artery disease [16]. Recently, results from a study suggest that the serum concentration of mitochondrial ATP synthase inhibitory factor 1 is independently negatively associated with all-cause and cardiovascular mortality in subjects with coronary artery disease [17], and another study suggest ectopic ATP synthase on endothelial cells is considered as a potential and novel therapeutic target for coronary artery disease [18].

\section{Limitations}

This study had several limitations. Firstly, the sample size was too small, the BTBD7_hsa_circ_000563 expression based on RT-qPCR and proteome-wide identification based on LC-MS/MS analysis have been conducted at 8 coronary artery segments from one subject; Secondly, the present study only explored the association of proteome-wide identification of BTBD7_hsa_circ_000563-regulated protein in coronary artery segments, and the possible mechanism underlying the association between BTBD7_hsa_circ_000563 and regulated proteins in coronary artery has not been studied; Finally, the therapeutical value of present finding has not been applied to clinical practice due to the absence of the function and mechanism study of BTBD7_hsa_circ_000563. Therefore, the verification study, mechanism study, and function study are necessary in order for the CAD patients to benefit from the personalized medicine in the future.

\section{Conclusion}

The most relevant finding of this study is that coronary artery segments with severe atherosclerotic stenosis showed extremely low expression of the BTBD7_hsa_circ_000563, as compared with normal coronary artery segments. Furthermore, it was predicted that hsa-miR-155-5p, and hsa-miR-130a-3p are the targets of the BTBD7_hsa_circ_000563. In addition, by means of a proteomics approach, 49 proteins regulated by enhanced BTBD7_hsa_circ_000563 expression were identified in human coronary artery segments. Bioinformatic analyses revealed that a large number of differentially expressed proteins were located in mitochondrion and involved in citrate cycle (TCA cycle) pathway. The ATP synthase may be the hub of the regulatory networks of BTBD7_hsa_circ_000563 in coronary artery.

\section{Declarations}

Ethics approval and consent to participate: The coronary artery samples were obtained from two autopsy cases at department of human anatomy in Nanjing Medical University. Informed consent from the bereaved family was obtained for the research use only of samples and the autopsy was conducted according to the guideline of the university. The methods were performed in accordance with the approved guidelines, and all experimental protocols were approved by the ethics committee of the Nanjing Medical University and the First Affiliated Hospital of Nanjing Medical University.

Consent for publication: Informed consent from the bereaved family was obtained for the research use only of samples and written informed consent to publish this information was also obtained from study 
participants.

Availability of data and materials: The data would be supplied upon request. The datasets used and/or analysed during the current study available from the correspondence author.

Competing interests: There was not any conflict of interest existing in this manuscript.

Funding $₫$ This study is supported by the National Natural Science Foundation of China (grants 30400173, 30971257, 81170180 and 81970302) whose receiver is En-Zhi Jia and it is funded by the Priority Academic Program Development of Jiangsu Higher Education Institutions.

Authors' contributions: As a guarantor, Enzhi Jia conceived the study. Jia-Xin Chen*, Lei Hua* are responsible for designing the study and writing the draft. * Jia-Xin Chen and Lei Hua contributed equally to the paper

Yong-Jie Zhang, Jian-Liang Jin, Mu-Feng Gu, Zhi-Yuan Mao, Hai-Jian Sun enrolled participants and collected data under the supervision of Chen-Hui Zhao, Qiao-Wei Jia. Jing Zhang and Jin-Xia Yuan coordinated the study.

Acknowledgements $₫$ Not applicable

\section{References}

1. Global Status Report on noncommunicable diseases 2014 (2015). Available at: http://www.thehealthwell.info/node/831799(Accessed: 18th December 2015).

2. Zhou Q, Zhang Z, Bei Y, Li G, Wang T. Circular RNAs as Novel Biomarkers for Cardiovascular Diseases. Adv Exp Med Biol. 2018;1087:159-170

3. Altesha MA, Ni T, Khan A, Liu K, Zheng X. Circular RNA in cardiovascular disease. J Cell Physiol. 2018 Oct 20. doi: 10.1002/jcp.27384

4. Maass PG, Glažar P, Memczak S, Dittmar G, Hollfinger I, Schreyer L, Sauer AV, Toka O, Aiuti A, Luft FC, Rajewsky N. A map of human circular RNAs in clinically relevant tissues. J Mol Med (Berl). 2017 Nov;95(11):1179-1189

5. Memczak S, Jens M, Elefsinioti A, Torti F, Krueger J, Rybak A, Maier L, Mackowiak SD, Gregersen LH, Munschauer M, Loewer A, Ziebold U, Landthaler M, Kocks C, le Noble F, Rajewsky N. Circular RNAs are a large class of animal RNAs with regulatory potency. Nature. 2013 Mar 21;495(7441):333-8

6. Rybak-Wolf A, Stottmeister C, Glažar P, Jens M, Pino N, Giusti S, Hanan M, Behm M, Bartok O, AshwalFluss R, Herzog M, Schreyer L, Papavasileiou P, Ivanov A, Öhman M(4), Refojo D, Kadener S, Rajewsky N. Circular RNAs in the Mammalian Brain Are Highly Abundant, Conserved, and Dynamically Expressed. Mol Cell. 2015 Jun 4;58(5):870-85

7. Stary HC. Natural history and histological classification of atherosclerotic lesions: an update. Arterioscler Thromb Vasc Biol. 2000 May;20(5):1177-8 
8. Qiao-Wei Jia, Zhao-Hong Chen, Xiao-Qing Ding, Jie-Yin Liu, Peng-Cheng Ge, Feng-Hui An, Li-Hua Li, Lian-Sheng Wang, Wen-Zhu Ma, Zhi-Jian Yang, and En-Zhi Jia. Predictive Effects of Circulating miR221,miR-130a and miR-155 for Coronary Heart Disease: A Multi-Ethnic Study in China. Cell Physiol Biochem 2017;42:808-823

9. Schmittgen TD, Livak KJ. Analyzing real-time PCR data by the comparative C(T) method. Nat Protoc. 2008;3:1101-1108

10. Szklarczyk D, Franceschini A, Wyder S, Forslund K, Heller D, Huerta-Cepas J, Simonovic M, Roth A, Santos A, Tsafou KP, Kuhn M, Bork P, Jensen LJ, von Mering C. STRING v10: protein-protein interaction networks, integrated over the tree of life. Nucleic Acids Res. 2015 Jan;43(Database issue):D447-5

11. Salzman J, Chen RE, Olsen MN, Wang PL, Brown PO. Cell-type specific features of circular RNA expression. PLoS Genet. 2013;9(9):e1003777

12. Suomalainen A, Battersby BJ. Mitochondrial diseases: the contribution of organelle stress responses to pathology. Nat Rev Mol Cell Biol 2017; 19: 77-92

13. Gorman GS, Chinnery PF, DiMauro S, et al. Mitochondrial diseases. Nat Rev Dis Primers 2016; $2: 16080$.

14. Calvo SE, Clauser KR, Mootha VK. MitoCarta2.0: an updated inventory of mammalian mitochondrial proteins. Nucleic Acids Res 2016; 44: D1251-57

15. Chandel NS. Mitochondria as signaling organelles. BMC Biol 2014; 12: 34

16. Chistiakov DA, Shkurat TP, Melnichenko AA, Grechko AV, Orekhov AN. The role of mitochondrial dysfunction in cardiovascular disease: a brief review. Ann Med. 2018 Mar;50(2):121-127

17. Maierean S, Serban MC, Rizzo M, Lippi G, Sahebkar A, Banach M. The potential role of mitochondrial ATP synthase inhibitory factor 1 (IF1) in coronary heart disease: a literature review. Lipids Health Dis. 2017 Feb 7;16(1):35

18. Fu Y, Zhu Y. Ectopic ATP synthase in endothelial cells: a novel cardiovascular therapeutic target. Curr Pharm Des. 2010;16(37):4074-9

\section{Tables}

Table 1. Natural history and histological classification of atherosclerotic lesions of the coronary artery samples 


\begin{tabular}{|l|l|l|l|l|l|l|l|l|l|l|l|l|l|}
\hline Case1 & Age & Gender & LM & LAD- & LAD- & LAD- & LCX- & LCX- & LCX- & RCA- & RCA- & RCA-d \\
& & & & $p$ & $m$ & $d$ & $p$ & $m$ & $d$ & $p$ & $m$ & \\
Grade & 64 & Male & 3 & 4 & 3 & 4 & 4 & 4 & 3 & 4 & 4 & 4 \\
Stage & & & 4 & 3 & 3 & 3 & 4 & 3 & 3 & 4 & 3 & 3 \\
\hline Case2 & Age & Gender & LM & LAD- & LAD- & LAD- & LCX- & LCX- & LCX- & RCA- & RCA- & RCA-d \\
& & & & $p$ & $m$ & $d$ & $p$ & $m$ & $d$ & $p$ & $m$ & \\
Grade & 68 & Male & 3 & 3 & 3 & 1 & 1 & 1 & 1 & 1 & 3 & 1 \\
\hline Stage & & & 3 & 3 & 3 & 0 & 0 & 1 & 1 & 2 & 3 & 1 \\
\hline
\end{tabular}

LM, the left main trunk; LAD-p, proximal segment of the left anterior descending; LAD-m, midsegment of the left anterior descending; LAD-d, distal segment of the left anterior descending; LCX-p, proximal segment of the left circumflex; LCX-m, midsegment of the left circumflex; LCX-d, distal segment of the left circumflex; RCA-p, proximal segment of the right coronary artery; RCA-m, midsegment of the right coronary artery; RCA-d, distal segment of theright coronary artery;

Grade, 1: stenosis from 0 to 25\%; Grade, 2:stenosis from 26 to $50 \%$; Grade, 3: stenosis from 51 to $75 \%$; Grade, 4 :stenosis from 76 to $100 \%$

Stage, 0: normal tunica intima; Stage, 1: fatty streak tunica intima; Stage, 2: fibrous plaques tunica intima ;Stage, 3: atherosclerotic tunica intima;Stage, secondary affection tunica intima

Table 2. The BTBD7_hsa_circ_000563 expression in coronary artery segments 


\begin{tabular}{|c|c|c|c|c|c|c|}
\hline Segment & h-actin & ct & $\triangle \mathrm{ct}$ & $\triangle \triangle \mathrm{ct}$ & $2^{\wedge}-\triangle \triangle \mathrm{ct}$ & Expression level \\
\hline \multirow{3}{*}{ LM } & 20.15 & 33.85 & 13.7 & 2.08 & 0.236514412 & \multirow[t]{3}{*}{0.1705} \\
\hline & 20.16 & 35.04 & 14.88 & 3.26 & 0.10438599 & \\
\hline & 20.18 & 31.87 & 11.69 & 0.07 & 0.952637998 & \\
\hline \multirow[t]{3}{*}{ LAD-p } & 20.06 & 31.41 & 11.35 & -0.27 & 1.205807828 & \multirow[t]{3}{*}{0.013726} \\
\hline & 19.85 & 37.38 & 17.53 & 5.91 & 0.016630784 & \\
\hline & 20.06 & 38.21 & 18.15 & 6.53 & 0.010821168 & \\
\hline \multirow[t]{3}{*}{ LAD-m } & 20.17 & 37.29 & 17.12 & 5.5 & 0.022097087 & \multirow[t]{3}{*}{1.325879} \\
\hline & 20.17 & 31.46 & 11.29 & -0.33 & 1.257013375 & \\
\hline & 20.19 & 31.33 & 11.14 & -0.48 & 1.394743666 & \\
\hline \multirow[t]{3}{*}{ LAD-d } & 19.14 & 30.86 & 11.72 & 0 & 1 & \multirow[t]{3}{*}{1} \\
\hline & 19.17 & 29.8 & 10.63 & 0 & 1 & \\
\hline & 18.91 & 31.42 & 12.51 & 0 & 1 & \\
\hline \multirow[t]{3}{*}{ LCX-p } & 18.85 & 28.19 & 9.34 & -2.28 & 4.856779538 & \multirow[t]{3}{*}{3.99756} \\
\hline & 18.98 & 30.86 & 11.88 & 0.26 & 0.835087919 & \\
\hline & 19.07 & 29.04 & 9.97 & -1.65 & 3.138336392 & \\
\hline \multirow[t]{3}{*}{ LCX-d } & 20.18 & 29.35 & 9.17 & -2.45 & 5.464161027 & \multirow[t]{3}{*}{5.31411} \\
\hline & 20.11 & 30.09 & 9.98 & -1.64 & 3.116658319 & \\
\hline & 20.26 & 29 & 8.74 & -2.88 & 7.361501205 & \\
\hline \multirow[t]{3}{*}{ RCA-p } & 20.11 & 30.08 & 9.97 & -1.65 & 3.138336392 & \multirow[t]{3}{*}{2.28259} \\
\hline & 19.94 & 30.5 & 10.56 & -1.06 & 2.084931522 & \\
\hline & 20.02 & 30.94 & 10.92 & -0.7 & 1.624504793 & \\
\hline \multirow[t]{3}{*}{ LCX-m } & 20.39 & 34.04 & 13.65 & 2.03 & 0.244855074 & \multirow[t]{3}{*}{2.15609} \\
\hline & 20.38 & 31.45 & 11.07 & -0.55 & 1.464085696 & \\
\hline & 20.22 & 30.33 & 10.11 & -1.51 & 2.848100391 & \\
\hline
\end{tabular}

LM, the left main trunk; LAD-p, proximal segment of the left anterior descending; LAD-m, midsegment of the left anterior descending; LAD- $d$, distal segment of the left anterior descending; LCX-p, proximal segment of the left circumflex; LCX-m, midsegment of the left circumflex; LCX-d, distal segment of the left circumflex; RCA-p, proximal segment of the right coronary artery

Table 3. The screening of significant difference proteins according to the BTBD7_hsa_circ_000563 expression level 


\begin{tabular}{|c|c|c|c|}
\hline Protein ID & Express trend & Protein ID & Express trend \\
\hline sp|P00367|DHE3_HUMAN & UP & sp|A0A075B6I0|LV861_HUMAN & DOWN \\
\hline sp|Q92552|RT27 HUMAN & ÜP & sp|P07954|FUMH_HUMAN & UP \\
\hline sp|P13861|KAP2 HÜMAN & ÜP & sp|Q02985|FHR3 HÜMAN & DOWN \\
\hline sp|O75746|CMC1 HUMAN & UP & sp|Q9HA77|SYCM HUMAN & UP \\
\hline sp|Q9UJYY1|HSPB8 HUMAN & UP & sp|O75208|COQ9 HUMAN & UP \\
\hline sp|P48735|IIDHP HÜMAN & "ÜP" & 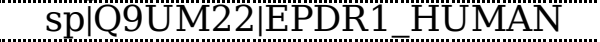 & "ÜP" \\
\hline sp|096000|NDUBA HUMAN & ÜР & sp|Q9Y6W5|WASF2 HUMAN & ÜP \\
\hline sp|P13671|CO6 HUUMAN & DOWN" & sp|PO9622|DLDH HUMAN & UP \\
\hline sp|Q99733|NP1L4 HUMAN & $\mathrm{UP}$ & sp|P48681|NEST"HUMANAN" & ÜP \\
\hline sp|P01624|KV315 HÜMAN & DÖWN & sp|Q16836|HCDH HUMANAN & UP \\
\hline sp|Q9Y235|ABEC2 HÜMAN & ÜP & sp|P01700|LV147 HÜMAN & DOWN \\
\hline sp|P16615]AT2A2 HUMAN & $\mathrm{UP}$ & sp|P36542|ATPG HUMAN & UP \\
\hline sp|P02760|AMBP HUMAN & DOWN" & sp|P06312|KV401 HUMAN" & DOWN" \\
\hline sp|P19022|CADH2 HUMAN & $\mathrm{UP}$ & sp|Q9NRPO|OSTC HUMAN & $\mathrm{UP}$ \\
\hline sp|P61626|LYSC HUÜMAN & DOWN & sp|P01701|LV151 HÜMAN & DOWN \\
\hline sp|O43837|IDH3B HUMAN & UP & sp|PO2 2511|CRYAB HUMAN & UP \\
\hline sp|Q9H479|FN3K HUMAN & UP & sp|Q15628|TRADD HUMAN & UP \\
\hline sp|O95831|AIFM1 HUMAN & ÜР & sp|P23786|CPT2 HUUMAN & ÜP \\
\hline sp|P17540|KCRS HUMAN & UP & sp|P00846|ATP6_HUMAN & UP' \\
\hline sp|P21912|SDHB HUMAN & ÜP & sp|O75367|H2AY HUMAN" & UP \\
\hline sp|P50213|IDH3A HUMAN & UP & sp|P12235|ADT1 HUMAN & UP \\
\hline sp|P26440|IVD HUMAN & $\mathrm{UP}$ & sp|P08559|ODPA HUMAN & UP \\
\hline sp|Q9Y287|ITM2B HUMAN & DOWN & sp|P42765|THIM_HUMAN & UP' \\
\hline sp|Q99798|ACON HÜMAN" & ÜP & sp|P30046|DOPD_HUMAN & UP \\
\hline sp|P46777|RL5_ḦUMAN & ÜР & & \\
\hline
\end{tabular}

\section{Abbreviations}

Coronary artery disease,CAD; Cardiovascular disease,CVD; Left anterior descending,LAD; Left circumflex,LCX; Right coronary artery,RCA; Left main trunk,LM; Haematoxylin and Eosin, H\&E; reverse transcription,RT; real-time quantitative (q) Polymerase Chain Reaction,RT-qPCR; Information Dependent Acquisition,IDA; false discovery rate,FDR; Ethylenediaminetetraacetic acid囚EDTA;

American Heart Association,AHA; MicroRNA,MiR; liquid chromatograph-mass spectrometer,LC-MS; Triethylamine borane,TEAB;

\section{Figures}




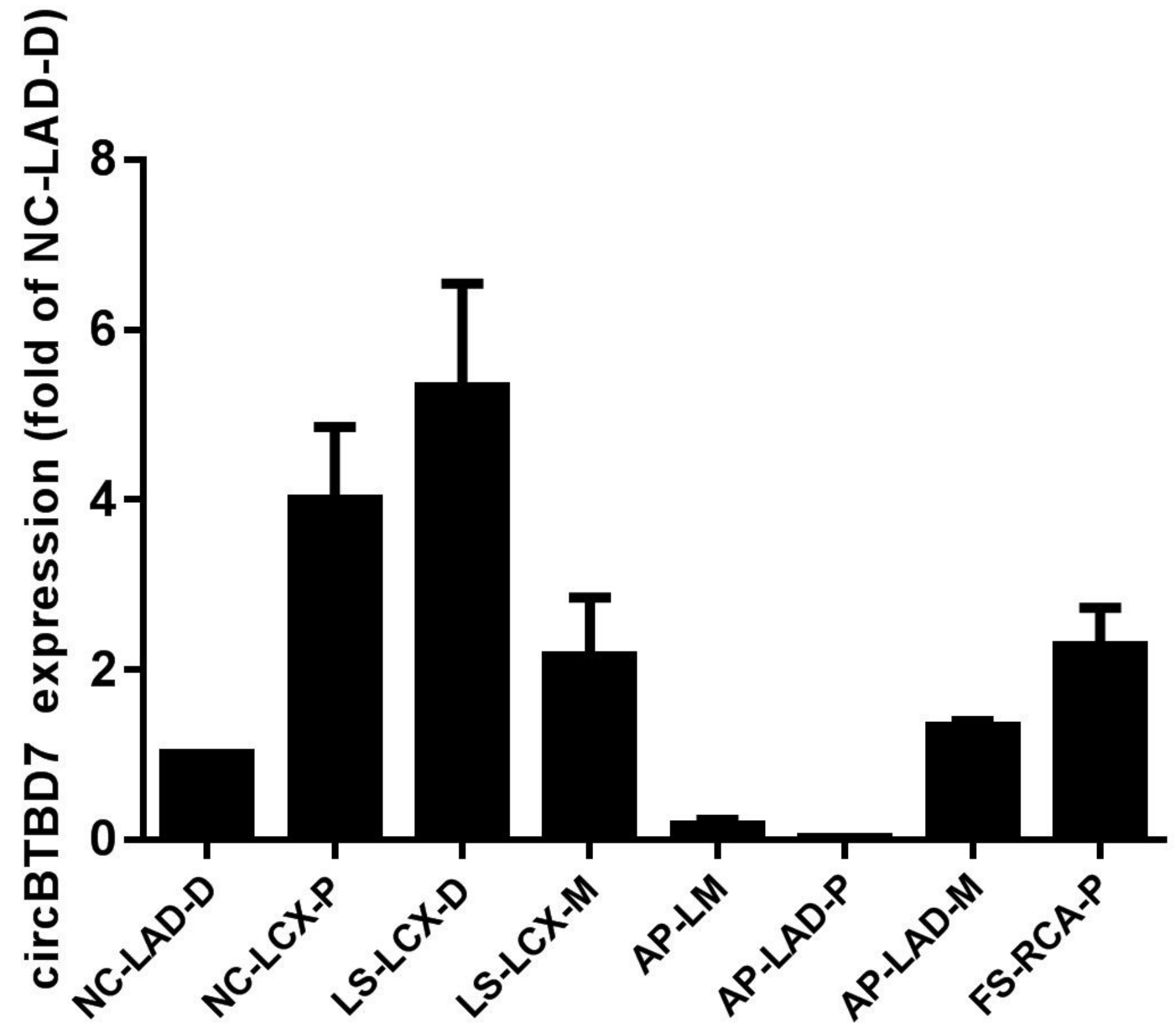

Figure 1

The BTBD7_hsa_circ_000563 expression in coronary artery segments 


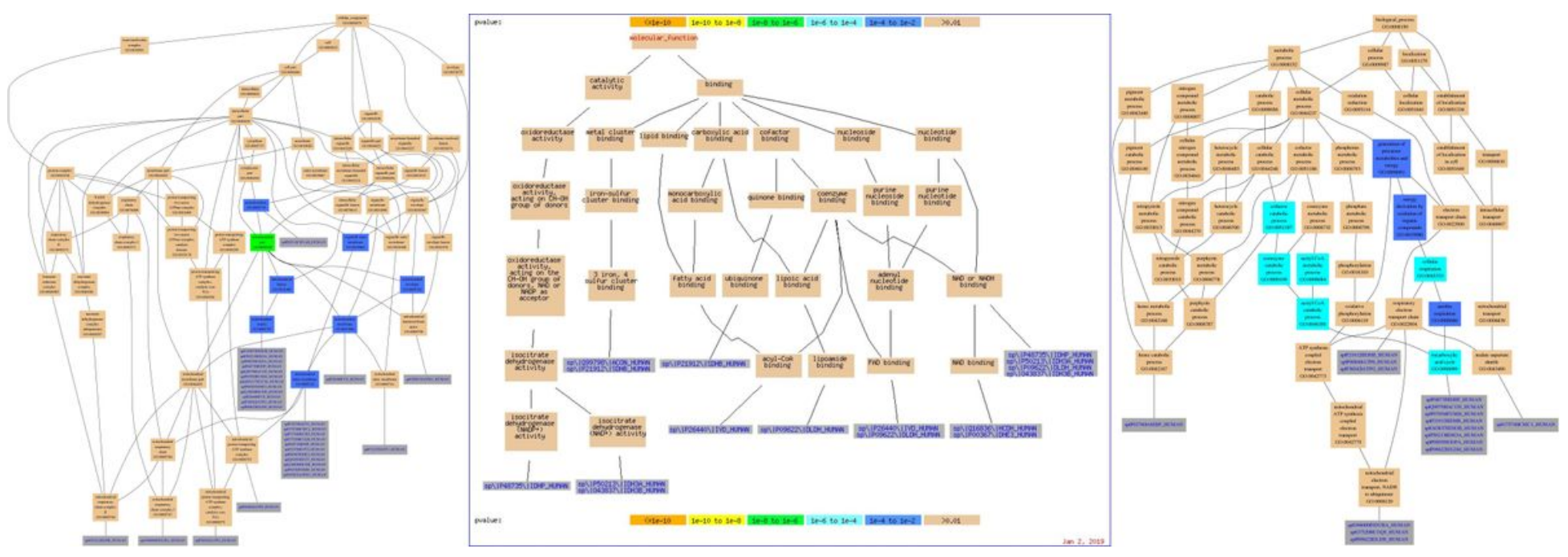

Figure 2

GO annotation of the differentially expressed proteins Panel (a): Cellular compartment annotation analysis for the differentially expressed proteins Panel (b): Molecular function annotation analysis for the differentially expressed proteins Panel (c): Biological process annotation analysis for the differentially expressed proteins

Parkinson's disease

Alzheimer's disease

Fatty acid metabolism

Primary immunodeficiency

D-Glutamine and D-glutamate metabolism

Valine, leucine and isoleucine degradation

Fatty acid elongation in mitochondria

Metabolic pathways

Apoptosis

Microbial metabolism in diverse environments

Citrate cycle (TCA cycle)

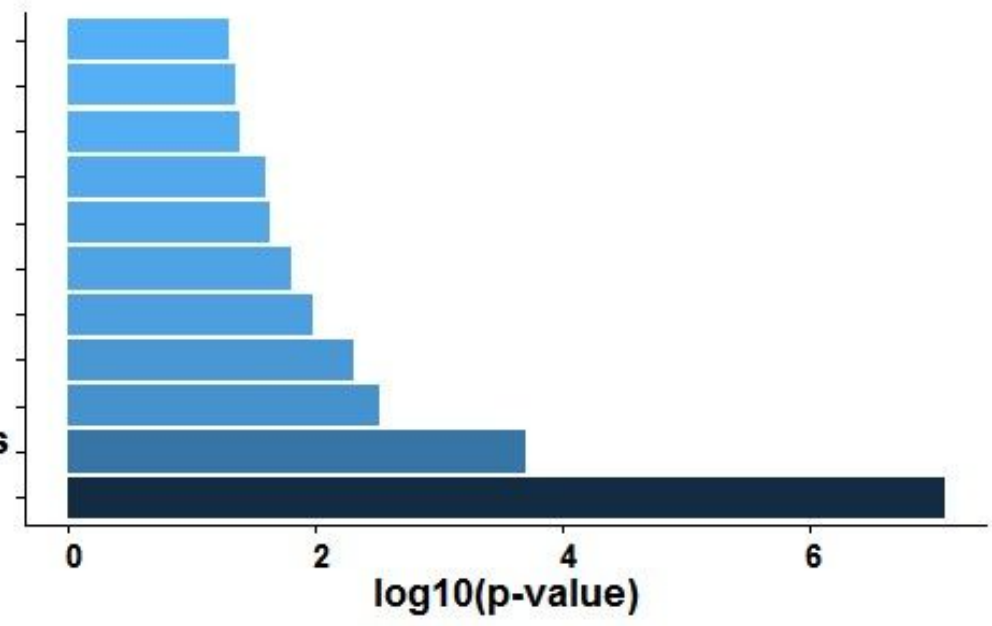

Figure 3

KEGG enrichment of the Differentially Expressed Proteins 

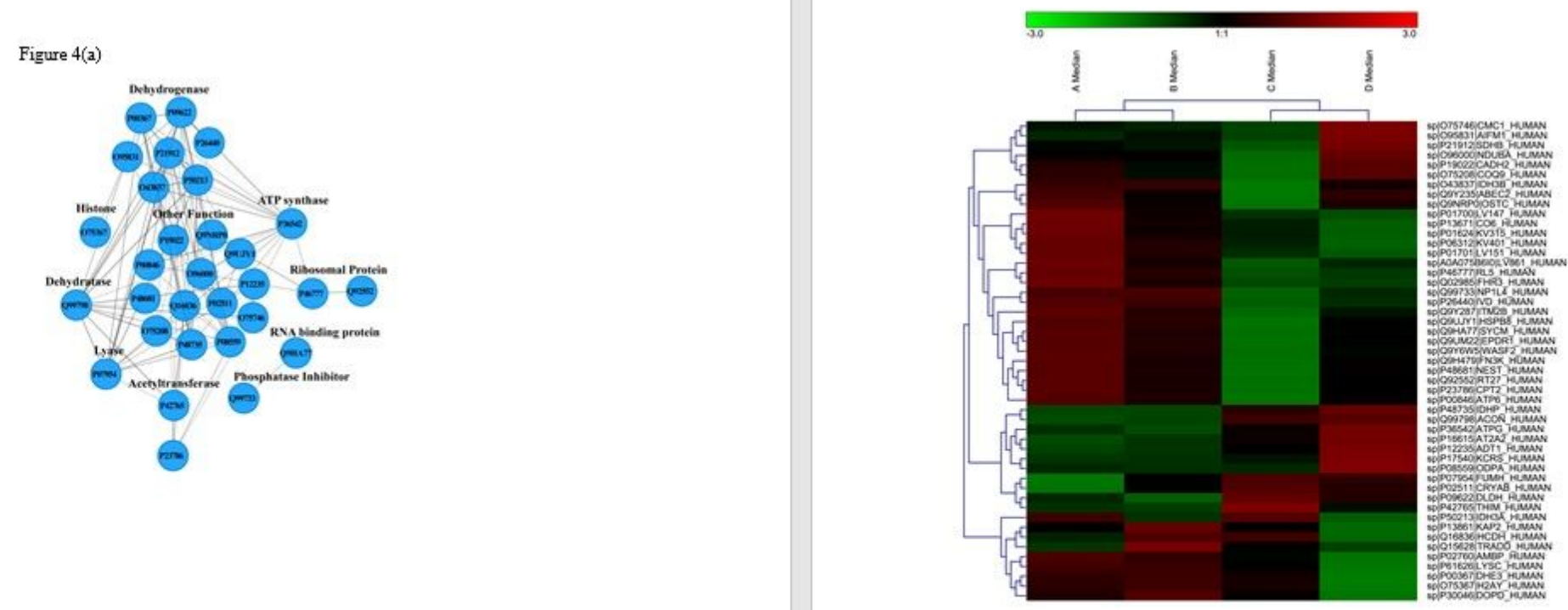

b

Figure 4

(a): Protein-protein interaction network of the Differentially Expressed Proteins Figure4(b):Heat map of the Differentially Expressed Proteins 